\title{
Modelado Matemático de la Transferencia de Calor del Proceso de Escaldado de Zanahoria (Daucus carota L.)
}

\author{
Fabián A. Ortega-Quintana*, Omar A. Pérez-Sierra, Liseth L. Tarrá-Lozano y Emiro A. López-Acosta \\ Universidad de Córdoba. Facultad de Ingenierías. Grupo de investigación GIPPAL. Carrera 6 No. 76-103. \\ Montería, Córdoba, Colombia. \\ (e-mail: fortega@correo.unicordoba.edu.co)
}

*Autor a quien debe ser dirigida la correspondencia

Recibido Abr. 20, 2017; Aceptado Jun. 28, 2017; Versión final Ago. 3, 2017, Publicado Dic. 2017

\section{Resumen}

Los objetivos del presente trabajo fueron modelar el proceso de escaldado de zanahoria y determinar el coeficiente convectivo de transferencia de calor del proceso. El escaldado se llevó a cabo en un baño de agua con temperaturas de $70 \pm 1^{\circ} \mathrm{C}, 80 \pm 1^{\circ} \mathrm{C}$ y $90 \pm 1^{\circ} \mathrm{C}$. Las temperaturas se midieron utilizando termopares tipo J con transmisor NI-USB-TC01 unido a la interfaz LabVIEW-2012. Se desarrolló un modelo matemático de transferencia de calor en estado no estacionario el cual fue resuelto por el método de elementos finitos asistido por COMSOL Multiphysics 3.5. La zanahoria fue considerada de forma cónica y con dos capas concéntricas de propiedades térmicas diferentes. Los resultados obtenidos con COMSOL son similares a los valores de temperaturas experimentales. Además los valores de los coeficientes convectivos se encuentran entre 433 y $643 \mathrm{~W} / \mathrm{m}^{2 \circ} \mathrm{C}$. Se concluye que el modelo desarrollado predice la temperatura con errores menores al $5 \%$.

Palabras clave: escaldado; convección; ley de Fourier; elementos finitos; modelado

\section{Mathematical Modeling of Heat Transfer of Blanching Process of Carrot (Daucus Carota L.)}

\begin{abstract}
The objectives of this research were to model the blanching process of carrot and to determine the convective heat transfer coefficient of the process. Blanching is carried out in water at temperatures of $70 \pm 1^{\circ} \mathrm{C}, 80 \pm 1^{\circ} \mathrm{C}$ and $90 \pm 1^{\circ} \mathrm{C}$. The temperatures were measured using J-type thermocouples with a NI USBTC01 transmitter attached to the interface LabVIEW-2012. A mathematical model of heat transfer in unsteady state was developed, and was solved by the finite element method assisted by COMSOL Multiphysics 3.5. The carrot sample was considered like cone-shaped with two concentric layers of different thermal properties. The numerical results obtained with COMSOL are similar to the values of the experimental temperatures. Also the values of the convective heat transfer coefficients were between 433 and $643 \mathrm{~W} / \mathrm{m}^{2 \circ} \mathrm{C}$. It is concluded that the proposed model predicts the temperature with errors less than $5 \%$.
\end{abstract}

Keywords: blanching; convection; Fourier's law; finite elements; modeling 


\section{INTRODUCCIÓN}

El procesado térmico es uno de los métodos más seguros y utilizados para la conservación de los alimentos. El escaldado de frutas y vegetales es una etapa que precede a los procesos como pasteurización, esterilización, congelación, entre otros. Por otro lado, el escaldado puede ser usado para inactivar enzimas que son responsables del deterioro durante el almacenamiento del alimento. Los productos escaldados, antes de almacenar en congelación o antes del enlatado, son más estables en términos de color, sabor y olor, conservando la calidad durante periodos más largos comparados con los productos sin escaldar. No obstante, los tiempos de escaldado deben ser calculados rigurosamente con el fin de evitar la destrucción de componentes nutricionales (como las vitaminas) y evitar los cambios de textura y cambios de color indeseables por el consumidor (Gamboa-Santos et al., 2012; Jaiswal et al., 2012; Ruiz-Ojeda y Peñas, 2013; Iribe-Salazar et al., 2015; Jeevitha et al., 2015; Xiao et al., 2017; Wang et al., 2017).

La complejidad estructural y la irregularidad de la superficie de algunos alimentos, como el caso de la zanahoria que tiene forma cónica y dos capas con composiciones químicas diferentes, hace que el cálculo de los tiempos de procesado térmico sea muy complicado. Para simplificar este cálculo se realizan consideraciones espaciales que reducen el problema a una sola dimensión con aproximaciones a geometrías simples (cilindros, placas o esferas) que ya tienen solución analítica, además se asume que las propiedades térmicas son constantes en todo el material (propiedad de isotropía) e independientes de la temperatura. Lo anterior implica que los tiempos estimados de procesamiento térmico sean o muy bajos, sin lograr el objetivo del escaldado, o muy altos que dañen la calidad nutricional y el rechazo del consumidor, lo que generaría sobrecostos a la industria (Arboleda et al., 2010; Madera et al., 2017; Ziabakhsh et al., 2016).

Por otro lado, los problemas de transferencia de calor por conducción en estado no estacionario, en general, están regidos por la Ley de Fourier expresada en ecuaciones diferenciales parciales sujetas a ciertas condiciones iniciales y condiciones de frontera. En décadas pasadas resolver este tipo de ecuaciones tenía un grado de complejidad y dificultad muy alto, esta herencia ha permanecido en los ingenieros de procesos actuales, no obstante, los avances en la tecnología computacional han facilitado la programación de cálculos más complejos y con tiempos de cómputo más cortos, lo cual permite resolver los problemas de tratamientos térmicos con mejor acercamiento a la realidad (Erdoğdu, 2008).

En ese sentido, los elementos finitos, que aproximan las ecuaciones diferenciales de gobierno de un sistema continuo a un sistema discreto, son una alternativa eficiente para resolver los modelos complejos de transferencia de calor y para tal fin, la simulación numérica con hardware y software apropiados, resulta ser una herramienta valiosa que permite estimar la distribución espacial y la dinámica de las temperaturas en el producto, minimizando los procedimientos experimentales reduciendo costos y tiempos de desarrollo de los procesos (Iribe-Salazar et al., 2015). Esta herramienta fue usada por Madera et al. (2017), Santana et al. (2011), Augusto et al. (2010), Pinho y Cristianini (2006) y Pornchaloempong et al. (2003), para evaluar alimentos con geometría regular o irregular. Por lo tanto, el objetivo de este trabajo fue modelar el proceso de escaldado y determinar el coeficiente convectivo de transferencia de calor en el escaldado de zanahoria empleando el método de elementos finitos (MEF) en el dominio 3D sin considerar contracción volumétrica.

\section{METODOLOGÍA}

La metodología se presenta en seis subsecciones: preparación de muestras, tratamiento térmico, adquisición de datos de temperatura, modelo de transferencia de calor, procedimiento de simulación y, por último, cálculo del coeficiente convectivo de transferencia de calor.

\section{Preparación de muestras}

Las zanahorias (Daucus carota L.) frescas fueron obtenidas en un mercado local en la ciudad de Montería, Colombia, mantenidas bajo refrigeración a $4^{\circ} \mathrm{C}$ y usadas en los experimentos dentro de las 24 horas siguientes. Se utilizaron cortes de zanahoria en forma de cono truncado, como se muestra en la figura 1 , con longitudes características cuyos valores se muestran en la tabla 1. Para el estudio térmico, las muestras de zanahoria se dividieron en dos partes: corazón y corteza, mostradas en la figura 2.

Tratamiento térmico

El procesamiento térmico de las muestras se llevó a cabo en un baño de agua con control On-Off de $\pm 1^{\circ} \mathrm{C}$ (modelo TECTRONBIO-20, Instrumentación Científica Técnica S.L.). El proceso de escaldado fue diseñado para temperaturas de agua de calentamiento de $70^{\circ} \mathrm{C}, 80^{\circ} \mathrm{C}$ y $90^{\circ} \mathrm{C}$ y un tiempo de proceso en el cual se logró el valor constante de la temperatura del centro geométrico. Finalmente, se escaldó una muestra de zanahoria a $85^{\circ} \mathrm{C}$ con el fin de verificar la validez del modelo. 
Tabla 1: Medidas de las dimensiones características.

\begin{tabular}{|c|c|}
\hline Dimensión & Valor $(\mathrm{cm}) \pm$ Desviación estándar \\
\hline $\mathrm{D}_{2}$ & $3,94 \pm 0,1$ \\
\hline $\mathrm{D}_{1}$ & $2,66 \pm 0,1$ \\
\hline $\mathrm{d}_{2}$ & $2,04 \pm 0,1$ \\
\hline $\mathrm{d}_{1}$ & $1,34 \pm 0,1$ \\
\hline $\mathrm{L}$ & $6,00 \pm 0,1$ \\
\hline
\end{tabular}

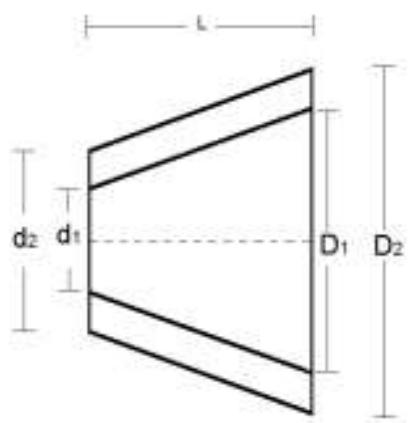

Fig. 1: Dimensiones características de las muestras de zanahoria.

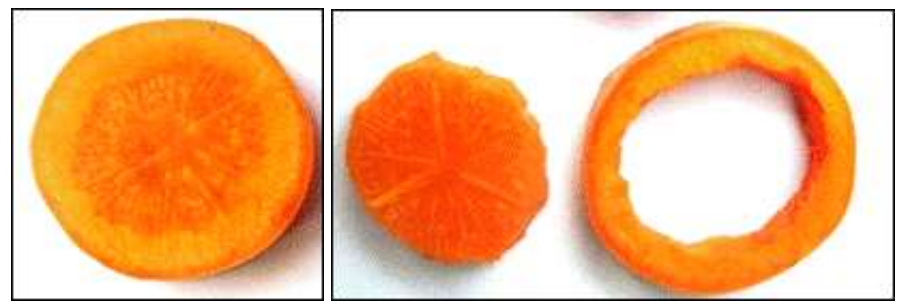

Fig. 2: Corte transversal de la zanahoria (cuadro izquierdo) y partes de la zanahoria para el estudio (corazón y corteza), (cuadro derecho).

Adquisición de datos de temperatura

Las temperaturas (del agua de escaldado, del centro geométrico y de la zona interfacial central entre las dos partes de la zanahoria) se midieron cada segundo utilizando termopares tipo $\mathrm{J}$ con transmisor de referencia NI USB-TC01 (National Instrument, USA). El registro de las temperaturas se obtuvo utilizando la interfaz LabVIEW. La figura 3 muestra los puntos donde se ubicaron los termopares en las muestras de zanahoria.

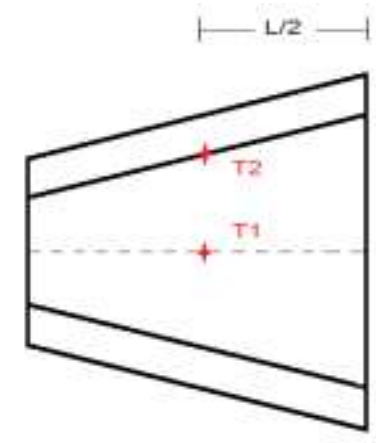

Fig. 3: Ubicación de los termopares en las muestras de zanahoria: centro geométrico (T1) e interfase (T2).

\section{Modelo de Transferencia de Calor}

Un modelo matemático fue desarrollado para describir el proceso de escaldado de la zanahoria. El modelo se obtuvo por un balance de energía térmica en estado no estacionario basado en la Ley de Fourier de la transferencia de calor teniendo en cuenta los siguientes supuestos: radiación térmica despreciable, propiedades físicas variables con la temperatura en cada punto del sólido y dependientes de la composición de la zanahoria, cambios despreciables en el volumen del sólido, transferencia de materia despreciable entre el agua y la zanahoria, la resistencia por contacto a la transferencia de calor entre las dos capas (corazón y corteza) es despreciable, por último, se considera la zanahoria de forma cónica perfecta con dos capas o partes concéntricas. El modelo es expresado en las ecns. (1)-(6): 


\section{Corazón:}

$\rho_{1} C_{p 1} \frac{\partial T}{\partial t}=\nabla\left(k_{1} \nabla T\right)$

\section{Corteza:}

$\rho_{2} C_{p 2} \frac{\partial T}{\partial t}=\nabla\left(k_{2} \nabla T\right)$

Condición en la interfase:

$\nabla\left(k_{1} \nabla T\right)=\nabla\left(k_{2} \nabla T\right)$

Condición inicial:

$T_{(\mathrm{x}, \mathrm{y}, \mathrm{z}, \mathrm{t}=0)}=T_{0}$

Condición de simetría:

$\frac{\partial T}{\partial x}=\frac{\partial T}{\partial y}=\frac{\partial T}{\partial z}=0$ para $x=0, y=0, z=0$

Condición de frontera (esta condición se cumple para toda la superficie expuesta al agua de calentamiento):

$-\left.k_{2} \frac{\partial T}{\partial n}\right|_{\Gamma}=h\left(T_{s}-T_{\infty}\right)$

Donde $n$ es el vector unitario normal hacia el exterior y $\Gamma$ es la superficie límite de la zanahoria.

Las propiedades termofísicas del corazón y corteza de las muestras de zanahoria se estimaron utilizando las correlaciones de Choi y Okos (1986). En la tabla 2 se muestra la composición porcentual promedio de las muestras de zanahoria obtenidas experimentalmente por los métodos de análisis indicados con un número superíndice: ${ }^{1934.06 / 96}$ de la A.O.A.C, ${ }^{2}$ Método Kjeldahl A.O.A.C/1984, ${ }^{3}$ extracción Soxhlet A.O.A.C/1984, ${ }^{4} 940.26 / 96$ de la A.O.A.C, ${ }^{5985.29 / 96}$ de la A.O.A.C, ${ }^{*}$ se obtuvo por diferencia entre el $100 \%$ y la suma de todos los componentes.

Tabla 2: Composición porcentual de la zanahoria.

\begin{tabular}{|l|c|c|}
\hline Composición $(\% p / p)$ & Corazón & Corteza \\
\hline Humedad $^{1}$ & $90,44 \pm 0,06$ & $88,15 \pm 0,04$ \\
\hline Proteína $^{2}$ & $1,11 \pm 0,08$ & $0,92 \pm 0,04$ \\
\hline Lípidos $^{3}$ & $0,19 \pm 0,02$ & $0,22 \pm 0,02$ \\
\hline Carbohidratos $^{*}$ & $5,84 \pm 0,05$ & $6,89 \pm 0,06$ \\
\hline Cenizas $^{4}$ & $0,75 \pm 0,01$ & $0,97 \pm 0,02$ \\
\hline Fibra $^{5}$ & $1,67 \pm 0,01$ & $2,85 \pm 0,01$ \\
\hline
\end{tabular}

Procedimiento de simulación

El modelo desarrollado fue resuelto por el método de elementos finitos usando el software COMSOL Multiphysics ${ }^{\circledR}$ 3.5. La malla fija fue creada automáticamente por el software y estaba compuesta por 48907 elementos tetraédricos distorsionados para aproximar mejor la curvatura del contorno irregular del dominio.

Cálculo del coeficiente convectivo de transferencia de calor

La determinación de valores de $h$ se llevó a cabo resolviendo un problema de transferencia de calor inverso, es decir, mediante la medición de la historia térmica interna de un cuerpo específico y luego, la solución de las ecuaciones de transferencia de calor. El problema de optimización se resolvió siguiendo el procedimiento propuesto por Duarte y Cristianini (2011). Para el cálculo del coeficiente convectivo de transferencia de calor se planteó el siguiente problema de optimización: 
1) Se declaró la Función Objetivo: se utilizó el error porcentual absoluto medio, el cual se minimiza (sugerido por Lespinard et al. (2009) y Duarte y Cristianini (2011).

$\left|\% \varepsilon_{\text {medio }}\right|=\frac{100}{m} \sum_{i=1}^{m} \frac{\left|T_{s}-T_{e}\right|}{T_{e}}$

Donde $T_{s}$ es la temperatura simulada, $T_{e}$ es la temperatura experimental, $\varepsilon_{\text {medio }}$ es el error porcentual absoluto medio y $m$ es el número de datos de temperaturas experimentales.

2) La variable de decisión fue el coeficiente convectivo de transferencia de calor $(h)$.

3) Las restricciones del problema de optimización fueron: i) Las ecuaciones del modelo matemático, ecns. (1 a 6); y ii) Las ecuaciones planteadas por Choi y Okos (1986) para calcular las propiedades termofísicas del centro y la corteza de la zanahoria.

\section{RESULTADOS Y DISCUSIÓN}

La comparación de los resultados experimentales y simulación son presentados en la figura 4 . Se observa que los resultados de temperatura numéricos obtenidos desde COMSOL están de acuerdo con los valores de las temperaturas experimentales. Además, se observan las tres etapas que presenta la temperatura de un centro térmico cuando se calienta, es decir, una etapa inicial donde la temperatura no tiene cambios apreciables debido a que el frente de calor no ha llegado a ese punto, una etapa intermedia donde ocurre un aumento de la temperatura de forma exponencial y, finalmente, la etapa donde se alcanza el equilibrio térmico entre el fluido y la zanahoria. Estas tres etapas presentadas por los datos experimentales son descritas de igual manera cuando se resuelve el modelo matemático planteado. Esto evidencia que el modelo planteado posee en su estructura los fenómenos más influyentes del proceso de escaldado, los cuales son la conducción y la convección de transferencia de calor, como lo muestra las ecns. (1)-(6). La tabla 3 muestra el error porcentual absoluto medio cuyos valores son menores del $5 \%$, lo cual demuestra la validez del modelo planteado.

Tabla 3: Coeficientes convectivos promedios y error porcentual absoluto medio.

\begin{tabular}{|c|c|c|}
\hline$T_{\infty}\left({ }^{\circ} \mathrm{C}\right)$ & $h\left(\mathrm{~W} / \mathrm{m}^{2}{ }^{\circ} \mathrm{C}\right)$ & Error porcentual absoluto medio \\
\hline 70 & $643 \pm 25$ & $2,85 \pm 0,94$ \\
\hline 80 & $580 \pm 17$ & $3,02 \pm 0,61$ \\
\hline 90 & $433 \pm 29$ & $3,42 \pm 0,89$ \\
\hline
\end{tabular}
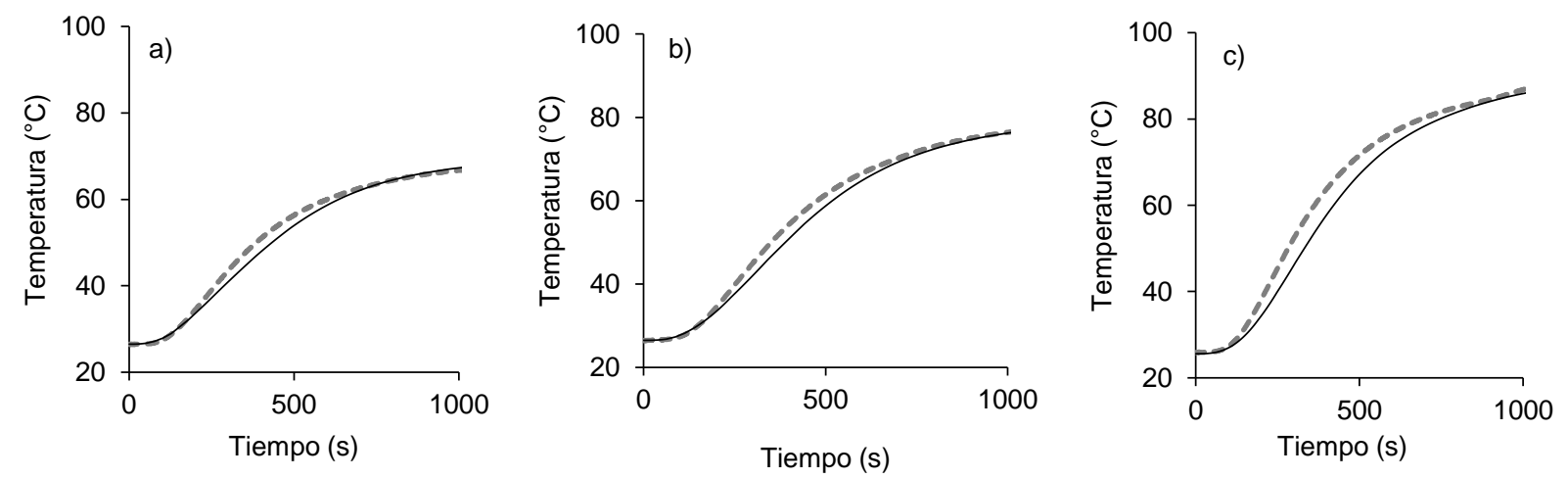

Fig. 4: Temperatura experimental (---) y simulada (-) del centro geométrico (T1) de la zanahoria para temperatura de agua de escaldado: a) $70^{\circ} \mathrm{C}$, b) $80^{\circ} \mathrm{C}$ y c) $90^{\circ} \mathrm{C}$.

Diferencias leves encontradas entre los datos experimentales y la simulación pueden ser atribuidas al controlador on-off del baño de agua de escaldado. Debido a este tipo de control, existe una variación en la temperatura del agua de escaldado a lo largo del proceso, dando como resultado un perfil de calentamiento en la muestra de zanahoria diferente al obtenido en una condición ideal predicho numéricamente. Situación semejante se presenta en el trabajo de Perussello et al. (2014). 
La figura 5 muestra la tendencia del coeficiente convectivo de transferencia de calor, el cual es decreciente con respecto a la temperatura del agua de escaldado. Anteriormente, se creía que el valor del coeficiente de transferencia de calor se incrementaba con el aumento de la temperatura del medio, pero el trabajo realizado por Lespinard et al. (2015) demuestra que para champiñones sometidos a escaldado asistido por ultrasonido a temperaturas entre $60-90^{\circ} \mathrm{C}$, el coeficiente de transferencia de calor disminuye desde 4044,79 hasta $2960,62 \mathrm{~W} / \mathrm{m}^{2 \circ} \mathrm{C}$, corroborando así el comportamiento de los valores obtenidos en esta investigación. Los valores del coeficiente convectivo de transferencia de calor encontrados son similares a los obtenidos por Lespinard et al. (2009) y Madera et al., (2017).

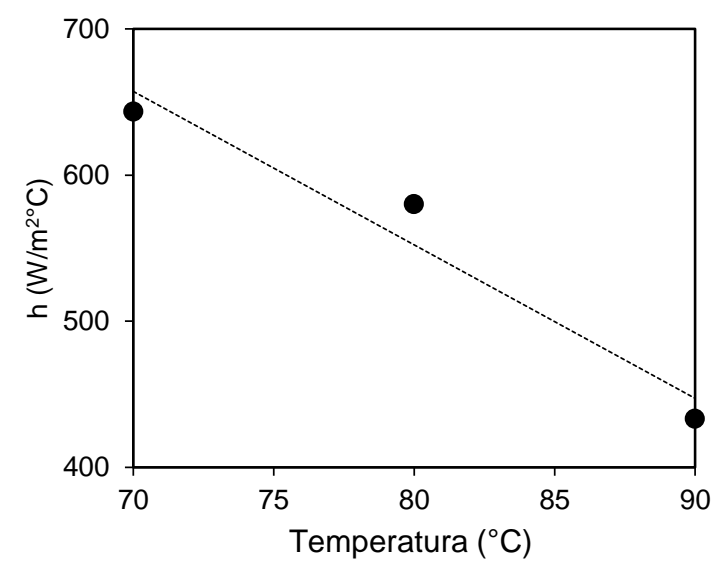

Fig. 5: Temperatura de escaldado versus $h$ promedio.

Por otro lado, la posición del punto frío o centro térmico de un alimento es crucial para el cálculo de un proceso térmico seguro, ya que, el punto frío es la localización de menos calentamiento dentro de un alimento, el cual recibe la menor letalidad de proceso acumulada (Fo). Por lo tanto, los cálculos del proceso deben estar basados en el perfil de temperatura-tiempo en el punto frío. La inexactitud de la ubicación del punto frío puede resultar en un procesado insuficiente (Pornchaloempong et al., 2003). En la figura 6 se muestra un gráfico 3D de la zanahoria presentando la distribución de la temperatura a los 10 minutos de escaldado a $70^{\circ} \mathrm{C}, 80^{\circ} \mathrm{C}$ y $90^{\circ} \mathrm{C}$ en dos planos, uno longitudinal y otro transversal (que pasa por el punto más frío). En esta figura se comprueba que el punto más frío se encuentra a $2 \mathrm{~cm}$ de la base más grande de la muestra de zanahoria, en el eje de rotación, es decir a 1/3 de fracción de la altura de la muestra desde la parte inferior de la misma, además las regiones que alcanzan mayores temperaturas son la parte inferior y superior de la zanahoria. Debido a que el centro térmico y centro geométrico difieren en ubicación dentro del sólido, no es recomendable realizar los cálculos de perfiles de temperatura y tiempos de escaldado suponiendo que la zanahoria es un cilindro finito perfecto e isotrópico.

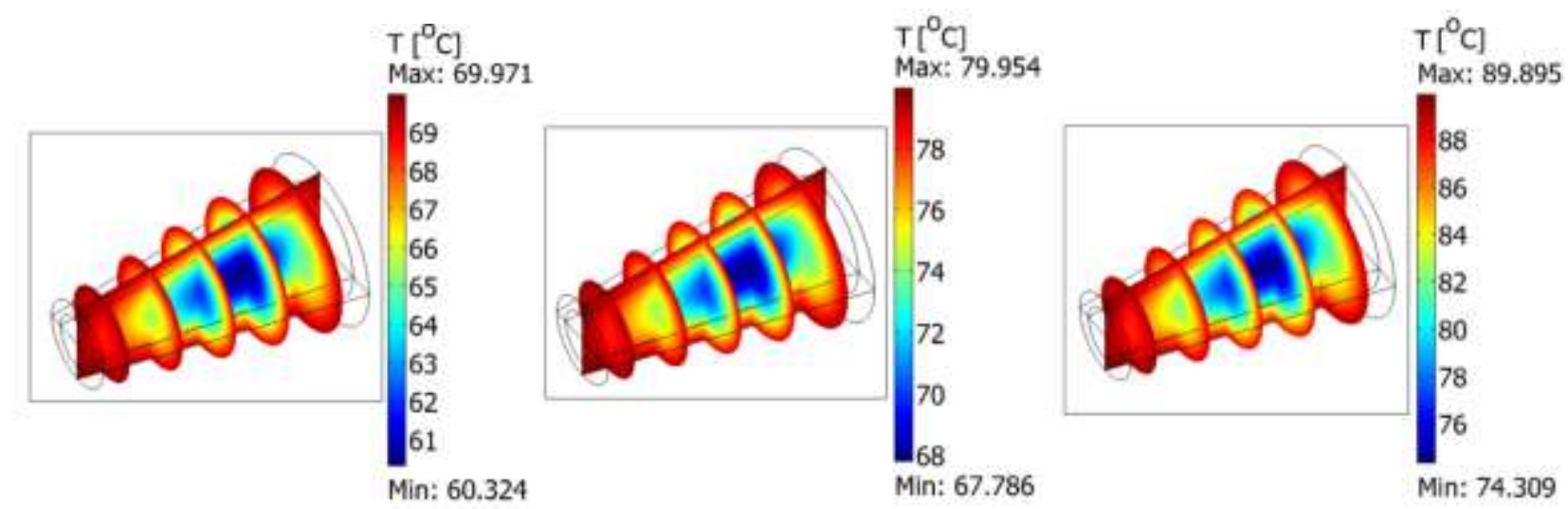

Fig. 6: Distribución de temperaturas a los 10 minutos de escaldado a $70^{\circ} \mathrm{C}, 80^{\circ} \mathrm{C}$ y $90^{\circ} \mathrm{C}$.

Finalmente, el modelo de transferencia de calor propuesto se evaluó para una temperatura de medio de calentamiento de $85^{\circ} \mathrm{C}$ y se midió la temperatura en el centro geométrico (T1) y en la interfase de las capas (T2). La figura 7 muestra los valores experimentales y simulados. El modelo matemático propuesto logra predecir los valores de la temperatura de la interfase con un error porcentual absoluto medio de $2,14 \%$ para el centro geométrico y $3,12 \%$ para la interfase, lo cual termina de corroborar que este modelo tiene una gran capacidad de predicción. 

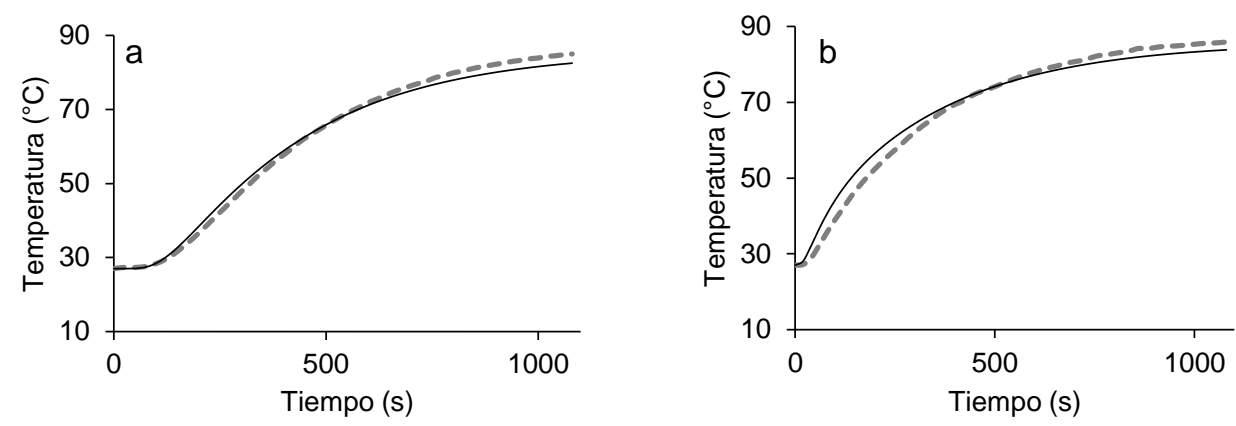

Fig. 7: Temperatura experimental (---) y simulada (-): a) Centro geométrico (T1) y b) Interfase (T2) de la zanahoria para temperatura de agua de escaldado $85^{\circ} \mathrm{C}$.

\section{CONCLUSIONES}

El modelo de transferencia de calor para el escaldado de zanahoria desarrollado en este estudio provee una predicción buena de la distribución de temperatura en la matriz alimenticia. Como los valores predichos desde el modelo coinciden estrechamente con los valores experimentales, la formulación matemática y los supuestos son considerados razonables para describir el proceso de escaldado de la zanahoria. Los valores del error porcentual absoluto medio menores al $5 \%$, demuestran, adicionalmente, la validez del modelo matemático de transferencia de calor. Este modelo representa una importante herramienta en el diseño y optimización de los procesos tanto que puede ser usado para probar nuevas condiciones experimentales, apuntando a la disminución de tiempos y costos involucrados en pruebas de laboratorio. Considerando que el modelo es basado en la fenomenología del proceso, puede ser utilizado para simular el escaldado de otros alimentos con las adaptaciones adecuadas, es decir, propiedades termofísicas específicas y coeficiente convectivo de transferencia de calor conocido.

\section{NOTACIÓN}

\section{Símbolos}

$C_{p 1} \quad$ calor específico del corazón de la zanahoria, $\mathrm{kJ} \mathrm{kg}^{-1}{ }^{\circ} \mathrm{C}^{-1}$

$C_{p 2}$ calor específico de la corteza de la zanahoria, $\mathrm{kJ} \mathrm{kg}^{-1}{ }^{\circ} \mathrm{C}^{-1}$

$k_{1} \quad$ conductividad térmica del corazón de la zanahoria, $\mathrm{Wm}^{-10} \mathrm{C}^{-1}$

$k_{2}$ conductividad térmica de la corteza de la zanahoria, $\mathrm{Wm}^{-1}{ }^{\circ} \mathrm{C}^{-1}$

$h$ coeficiente convectivo de transferencia de calor, $\mathrm{W} \mathrm{m}^{-2}{ }^{\circ} \mathrm{C}^{-1}$

$T_{\infty} \quad$ temperatura del agua de calentamiento, ${ }^{\circ} \mathrm{C}$

$T_{0} \quad$ temperatura inicial de la zanahoria, ${ }^{\circ} \mathrm{C}$

$T$ temperatura de la zanahoria a un tiempo $\mathrm{t},{ }^{\circ} \mathrm{C}$

$T_{S} \quad$ temperatura de la superficie de la zanahoria, ${ }^{\circ} \mathrm{C}$

\section{Símbolos griegos}

$\rho_{1} \quad$ densidad del corazón de la zanahoria, $\mathrm{kg} \mathrm{m}^{-3}$

$\rho_{2} \quad$ densidad de la corteza de la zanahoria, $\mathrm{kg} \mathrm{m}^{-3}$

\section{REFERENCIAS}

A.O.A.C., Official Methods of Analysis, The Association of Official Analytical Chemists International, Gaithersburg, USA (1984)

A.O.A.C., Official Methods of Analysis, The Association of Official Analytical Chemists, Washington, USA (1996)

Arboleda, D. y otros tres autores, Modelo matemático para el proceso térmico de productos cárnicos de geometría cilíndric, Revista DYNA, 77(164), 301-307 (2010)

Augusto, P. y M. Cristianini, Evaluation of geometric symmetry condition in numerical simulations of thermal process of packed liquid food by computational fluid dynamics (CFD), International Journal of Food Engineering, 6(5), art.1 (2010)

Choi, Y. y M. Okos, Effects of Temperature and Composition on the Thermal Properties of Foods, Journal of Food Process and Applications, 1(1), 93-101 (1986) 
Duarte, P. y M. Cristianini, Determining the Convective Heat Transfer Coefficient $(h)$ in Thermal Process of Foods, International Journal of Food Engineering, 7(4), art. 15 (2011)

Erdoğdu, F., A review on simultaneous determination of thermal diffusivity and heat transfer coefficient, Journal of Food Engineering, 86, 453-459 (2008)

Gamboa-Santos, J. y otros tres autores, Effects of conventional and ultrasound blanching on enzyme inactivation and carbohydrate content of carrots, European Food Research and Technology, 234, 1071-1079 (2012)

Iribe-Salazar, R. y otros seis autores, Heat Transfer during Blanching and Hydrocooling of Broccoli Florets, Journal of Food Science, 80(12), 2774-2781 (2015)

Jaiswal, A., S. Gupta y N. Abu-Ghannam, Kinetic evaluation of colour, texture, polyphenols and antioxidant capacity of Irish York cabbage after blanching treatment, Food Chemistry, 131(1), 63-72 (2012)

Jeevitha, G., H. Hebbar y K. Raghavarao, Modeling of Peroxidase Inactivation and Temperature Profile during Infrared Blanching of Red Bell Pepper, Journal of Food Processing and Preservation, 40(1), 83-93 (2015)

Lespinard, A. y otros cuatro autores, Effect of Ultrasonic-Assisted Blanching on Size Variation, Heat Transfer, and Quality Parameters of Mushrooms, Food Bioprocess Technology, 8, 41-53 (2015)

Lespinard, A. y otros tres autores, Experimental determination and modelling of size variation, heat transfer and quality indexes during mushroom blanching, Journal of Food Engineering, 92(1), 8-17 (2009)

Madera, S. y otros tres autores, Determinación del Coeficiente Convectivo de Transferencia de Calor del Proceso de Zapallo (Cucurbita máxima), Información Tecnológica 28(3), 59-66 (2017)

Perussello, C. y otros tres autores, Heat and mass transfer modeling of the osmo-convective drying of yacon roots (Smallanthus sonchifolius), Applied Thermal Engineering, 63, 23-32 (2014)

Pinho, C. y M. Cristianini, Método de otimização para determinação do coeficiente convectivo de transferência de calor em alimentos esterilizados em embalagem de vidro, Brazilian Journal of Food Technology, 9(3), 157-163 (2006)

Pornchaloempong, P., y otros tres autores, Numerical simulation of conduction heating in conically shaped bodies, Journal of Food Process and Engineering, 25, 539-550 (2003)

Ruiz-Ojeda, L. y F. Peñas, Comparison study of conventional hot-water and microwave blanching on quality of green beans, Innovative Food Science \& Emerging Technologies, 20, 191-197 (2013)

Santana, F., P. Augusto y M. Cristianini, Determination of the convective heat transfer Coefficient $(\mathrm{h})$ in the sterilization of retortable pouches, International Journal of Food Engineering, 7(1), art. 10 (2011)

Wang, J., y otros nueve autores, Effect of various blanching methods on weight loss, enzymes inactivation, phytochemical contentes, antioxidant capacity, ultrastructure and drying kinetics of red bell pepper (Capsicum annuum L.), LWT Food Science and Technology, 77, 337-347 (2017)

Xiao, H., y otros siete autores, Recent developments and trends in termal blanching-A comprehensive review, Information Processing in Agriculture, 4, 101-127 (2017)

Ziabakhsh, M., y otros cuatro autores, Effect of blanching on enzyme activity, color changes, anthocyanin, stability and extractability of mangosteen pericarp: A kinetic study, J. of Food Engineering, 178, $12-19$ (2016) 